\title{
Population-based SEER analysis of survival in colorectal cancer patients with or without resection of lung and liver metastases
}

\author{
Alexander R. Siebenhüner ${ }^{1 *}$ (D) Ulrich Güller ${ }^{2,3,4}$ and Rene Warschkow ${ }^{5,6}$
}

\begin{abstract}
Background: Approximately one third of all patients with CRC present with, or subsequently develop, colorectal liver metastases (CRLM). The objective of this population-based analysis was to assess the impact of resection of liver only, lung only and liver and lung metastases on survival in patients with metastatic colorectal cancer (mCRC) and resected primary tumor.
\end{abstract}

Methods: Ten thousand three hundred twenty-five patients diagnosed with mCRC between 2010 and 2015 with resected primary were identified in the Surveillance, Epidemiology and End Results (SEER) database. Overall, (OS) and cancer-specific survival (CSS) were analyzed by Cox regression with multivariable, inverse propensity weight, near far matching and propensity score adjustment.

Results: The majority (79.4\%) of patients had only liver metastases, $7.8 \%$ only lung metastases and $12.8 \%$ metastases of lung and liver. 3-year OS was 44.5 and $27.5 \%$ for patients with and without metastasectomy (HR=0.62, 95\% Cl: 0.58$0.65, P<0.001)$. Metastasectomy uniformly improved CSS in patients with liver metastases ( $\mathrm{HR}=0.72,95 \% \mathrm{Cl}: 0.67-0.77$, $P<0.001)$ but not in patients with lung metastases ( $H R=0.84,95 \% \mathrm{Cl}: 0.62-1.12, P=0.232)$ and combined liver and lung metastases ( $\mathrm{HR}=0.89,95 \% \mathrm{Cl}: 0.75-1.06, P=0.196)$ in multivariable analysis. Adjustment by inverse propensity weight, near far matching and propensity score and analysis of OS yielded similar results.

Conclusions: This is the first SEER analysis assessing the impact of metastasectomy in mCRC patients with removed primary tumor on survival. The analysis provides compelling evidence of a statistically significant and clinically relevant increase in OS and CSS for liver resection but not for metastasectomy of lung or both sites.

Keywords: Colorectal cancer, Right and left sided tumor, Liver metastasis, Lung metastasis, Chemotherapy, Surgery, Surveillance epidemiology and end results database (SEER), Propensity score analysis

\footnotetext{
* Correspondence: alexander.siebenhuener@usz.ch

${ }^{1}$ Clinic for Medical Oncology and Hematology, University Hospital Zurich and University of Zurich, CH-8091 Zürich, Switzerland

Full list of author information is available at the end of the article
}

(c) The Author(s). 2020 Open Access This article is licensed under a Creative Commons Attribution 4.0 International License, which permits use, sharing, adaptation, distribution and reproduction in any medium or format, as long as you give appropriate credit to the original author(s) and the source, provide a link to the Creative Commons licence, and indicate if changes were made. The images or other third party material in this article are included in the article's Creative Commons licence, unless indicated otherwise in a credit line to the material. If material is not included in the article's Creative Commons licence and your intended use is not permitted by statutory regulation or exceeds the permitted use, you will need to obtain permission directly from the copyright holder. To view a copy of this licence, visit http://creativecommons.org/licenses/by/4.0/. The Creative Commons Public Domain Dedication waiver (http://creativecommons.org/publicdomain/zero/1.0/) applies to the data made available in this article, unless otherwise stated in a credit line to the data. 


\section{Background}

Colorectal cancer (CRC) is one of the most frequent malignant tumors. Indeed, based on the latest update of the national cancer statistics at the United States, CRC has the third highest incidence in both men and in women [1]. The incidence rate is estimated to be 1.2 million per annum in the US, and more than 600,000 patients die from this cancer every year [2]. Approximately one third of all patients with CRC present with, or subsequently develop, colorectal liver metastases (CRLM). Moreover, another $23-38 \%$ of patients already have, or will develop extra-hepatic disease [3-5].

Over the past years the landscape of treatment modalities in patients with metastatic colorectal cancer has expanded tremendously and improved the median overall survival (OS) from a median of 5 months in 1993 [6] to more than 3 years nowadays [7]. Factors improving median survival rates of metastatic CRC are a better understanding of the heterogeneity of the disease based on rat sarcoma (RAS) and rapidly accelerated fibrosarcoma- (RAF) mutations as well as mismatch repair status, which allows a more patient-tailored treatment using antibody treatment in combination with chemotherapy or immunotherapy [8-12]. Moreover, the location of the primary cancer - left versus right hemicolon - has been recognized as important prognostic and predictive factor, particularly regarding the efficacy of epidermal growth factor receptor (EGFR) antibodies [13-16]. Despite this, rapidly accumulating knowledge about tumor heterogeneity of metastatic colorectal cancer $(\mathrm{mCRC})$, many relevant questions regarding treatment sequences as well the impact and timing of resection of lung and liver metastases remain. The latest versions of national and international guidelines include resection of metastases at some point in the treatment of mCRC. However, these recommendations are often vague $[17,18]$. While it is well recognized that liver resections in curative intent should be performed, there is ongoing debate regarding the resection of lung metastases and both lung and liver metastases.

Hence, the objective of the present populationbased analysis was to assess the impact of resection of liver only, lung only and liver and lung metastases on survival in patients with $\mathrm{mCRC}$ with resected primary tumor.

\section{Methods}

\section{Study cohort}

The 2015 custom text data-version of the Surveillance, Epidemiology, and End Results (SEER) Program of the National Cancer Institute in the United States, covering approximately $28 \%$ of cancer cases in the United States, was the source of the present population-based analysis [19].

\section{Study design}

All patients diagnosed with colorectal cancer between 2010 and 2015 were eligible for the analysis. Patients aged below 18 years, with missing diagnosis by histology, secondary malignancies preceding the $\mathrm{mCRC}$, other histology than adenocarcinoma, incomplete staging, nonmetastatic cancer, overlapping or unknown localization of the primary, metastasis other than liver and/or lung, undefined localization of the metastasis, not-resected primary, and unknown non - primary surgery or non primary surgery limited to distant lymph nodes were excluded. Figure 1 depicts the selection process.

\section{Statistical methods}

Statistical analyses were performed using the $\mathrm{R}$ statistical software (www.r-project.org). A two-sided $p$-value $<0.05$ was considered statistically significant. Cancer-specific (CSS) and overall survivals were the co-primary endpoints. $P$-values were estimated using likelihood-ratio tests. The proportional hazard assumption for Cox regression was tested by scaled Schoenfeld residuals and by inspection of the hazard ratio (HR) plots [20]. After descriptive analysis, the imbalances regarding prognostic factors between patients with and without metastasectomy were assessed by multivariable logistic regression with adjustment for the site of the primary, metastatic site (lung, liver, both), T-stage, nodal status, grading, chemotherapy [21], year of diagnosis, age, gender, ethnicity, and marital status (risk set). The impact of metastasectomy on survival was analyzed with and without adjustment for the risk set. The impact of metastasectomy on survival was further analyzed with inverse propensity weight adjustment (stabilized weights) using the "ipw" R package [22]. Thereafter an exact matched propensity score and weighted analysis was performed as a superior and more refined statistical method for adjustment [23-25] using the "MatchIt" $R$ package [26]. Each patient with metastasectomy was matched to all possible patients without with exactly the same values on all the covariates in the risk set, forming subclasses such that within each subclass both weighted groups have exactly the same covariate values. Patients without a counterpart among the patients in the other group were excluded from this analysis. Finally, a near-far matched analysis was performed to assess the impact of metastasectomy with adjustment for unobserved confounding variables. FIPS county codes were used as the instrumental variable to build an encouraged and discouraged group according to county codes with a high and low rate of metastasectomy. These two groups were then matched and analyzed in a paired Cox-regression model.

Thereafter, the entire analysis was repeated in each of the three groups according to the site of the metastasis 


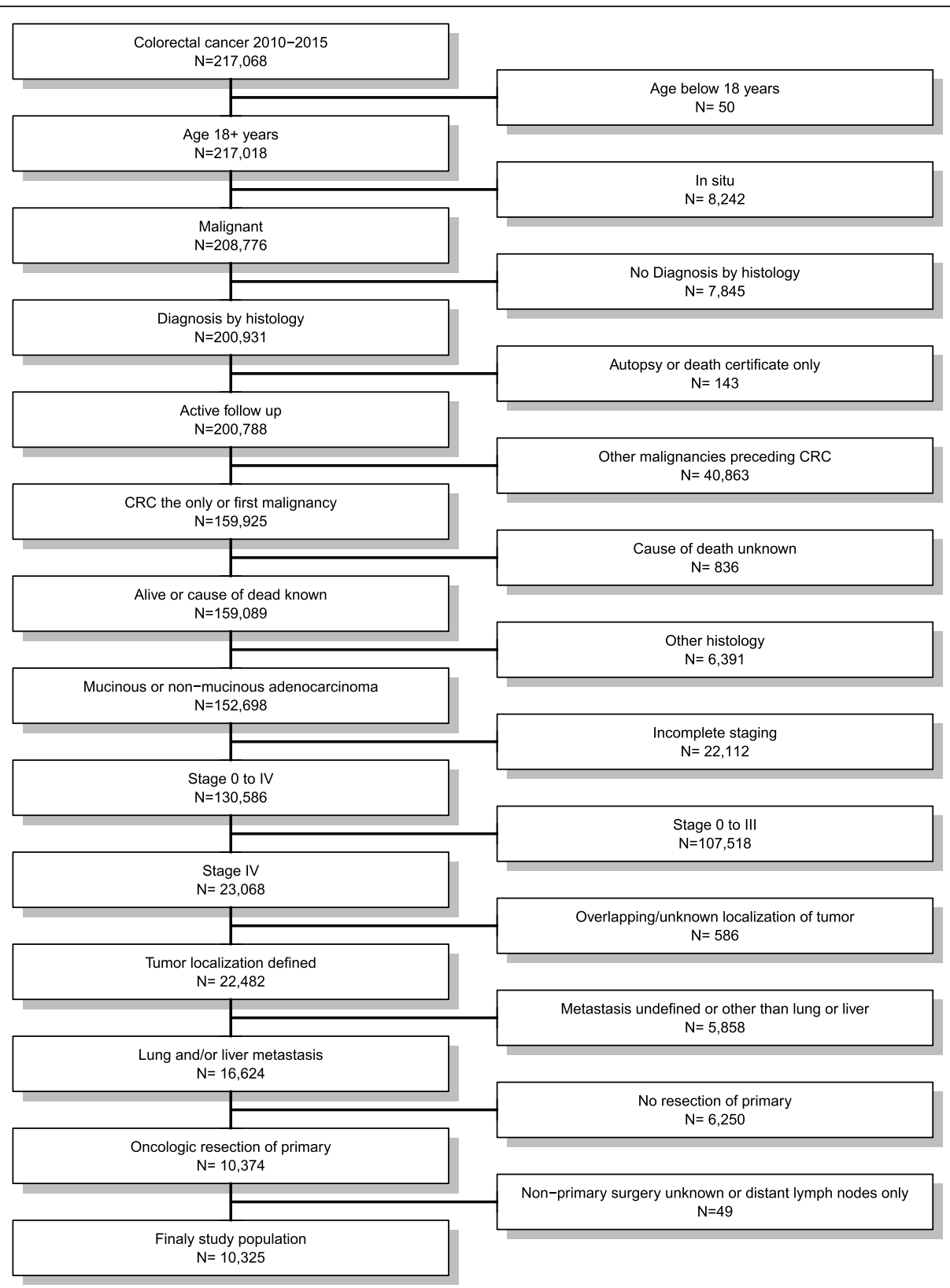

Fig. 1 Flow chart of patients' cohort definition. Data from the Surveillance, Epidemiology, and End Results (SEER) Program of the National Cancer Institute in the United States, covering approximately 28\% of cancer cases in the United States were used for the present population-based analysis. Of 217,068 patients diagnosed with colorectal cancer between 2010 and 2015, 10,325 were eligible for analysis at the end of the selection process

using the same risk set except from the site of the metastasis.

\section{Results}

\section{Patients characteristics}

Ten thousand three hundred twenty-five of 217,068 patients diagnosed with colorectal cancer between 2010 and 2015 were included (Fig. 1). The median follow-up time was 15 months (interquartile range: 6 to 28 months) for all patients and 19 months (interquartile range: 8 to 35 months) for those alive at the end of follow up. At the end of follow-up, 4299 (41.6\%) patients were alive, 5667 (54.9\%) died from cancer and 359 (3.5\%) from other reasons. Overall, 8195 (79.4\%) patients had liver metastases only, 807 (7.8\%) patients presented with lung metastases only and $1323(12.8 \%)$ presented with metastases on both sites (Table 1). Metastasectomy was performed in 2906 (28.1\%) patients. The multivariable 
Table 1 Patient characteristics

\begin{tabular}{|c|c|c|c|c|c|c|c|c|c|}
\hline & & \multirow{2}{*}{$\begin{array}{l}\text { Total } \\
N=10,325\end{array}$} & \multicolumn{3}{|l|}{ Resection } & \multicolumn{4}{|l|}{ Metastasis } \\
\hline & & & $\begin{array}{l}\text { No Resection } \\
N=7419\end{array}$ & $\begin{array}{l}\text { Resection } \\
N=2906\end{array}$ & $\mathrm{P}^{\mathrm{a}}$ & $\begin{array}{l}\text { Liver } \\
N=8195\end{array}$ & $\begin{array}{l}\text { Lung } \\
N=807\end{array}$ & $\begin{array}{l}\text { Both } \\
N=1323\end{array}$ & $\mathrm{P}^{\mathrm{a}}$ \\
\hline \multirow[t]{2}{*}{ Resection } & No & 7419 (71.9\%) & 7419 (100\%) & - & - & $5695(69.5 \%)$ & $663(82.2 \%)$ & 1061 (80.2\%) & $<0.001$ \\
\hline & Yes & 2906 (28.1\%) & - & 2906 (100\%) & & $2500(30.5 \%)$ & $144(17.8 \%)$ & $262(19.8 \%)$ & \\
\hline \multirow[t]{3}{*}{ Metastasis } & Liver only & 8195 (79.4\%) & 5695 (76.8\%) & $2500(86.0 \%)$ & $<0.001$ & 8195 (100\%) & - & - & - \\
\hline & Lung only & 807 (7.8\%) & $663(8.9 \%)$ & $144(5.0 \%)$ & & - & 807 (100\%) & - & \\
\hline & Liver and Lung & $1323(12.8 \%)$ & 1061 (14.3\%) & $262(9.0 \%)$ & & - & - & $1323(100 \%)$ & \\
\hline \multirow[t]{5}{*}{ Tumor localization } & Right colon & 3485 (33.8\%) & 2602 (35.1\%) & $883(30.4 \%)$ & $<0.001$ & 2808 (34.3\%) & $220(27.3 \%)$ & $457(34.5 \%)$ & $<0.001$ \\
\hline & Transverse & 1401 (13.6\%) & 1005 (13.5\%) & $396(13.6 \%)$ & & 1140 (13.9\%) & $98(12.1 \%)$ & $163(12.3 \%)$ & \\
\hline & Left colon & 3311 (32.1\%) & 2346 (31.6\%) & $965(33.2 \%)$ & & $2666(32.5 \%)$ & $231(28.6 \%)$ & $414(31.3 \%)$ & \\
\hline & Rectosigmoid & 966 (9.4\%) & $680(9.2 \%)$ & $286(9.8 \%)$ & & 736 (9.0\%) & $88(10.9 \%)$ & $142(10.7 \%)$ & \\
\hline & Rectum & $1162(11.3 \%)$ & 786 (10.6\%) & 376 (12.9\%) & & $845(10.3 \%)$ & $170(21.1 \%)$ & $147(11.1 \%)$ & \\
\hline \multirow[t]{2}{*}{ T-Stage } & $\mathrm{T} 1$ to $\mathrm{T} 3$ & $6643(64.3 \%)$ & 4727 (63.7\%) & 1916 (65.9\%) & 0.034 & $5348(65.3 \%)$ & $532(65.9 \%)$ & $763(57.7 \%)$ & $<0.001$ \\
\hline & $\mathrm{T} 4$ & 3682 (35.7\%) & 2692 (36.3\%) & $990(34.1 \%)$ & & 2847 (34.7\%) & 275 (34.1\%) & $560(42.3 \%)$ & \\
\hline \multirow[t]{2}{*}{ N-Stage } & No & 2103 (20.4\%) & 1439 (19.4\%) & $664(22.8 \%)$ & $<0.001$ & $1654(20.2 \%)$ & $230(28.5 \%)$ & $219(16.6 \%)$ & $<0.001$ \\
\hline & $\mathrm{N}+$ & 8222 (79.6\%) & 5980 (80.6\%) & 2242 (77.2\%) & & $6541(79.8 \%)$ & 577 (71.5\%) & 1104 (83.4\%) & \\
\hline \multirow[t]{2}{*}{ Grade } & $\mathrm{G} 1 / 2$ & 7715 (74.7\%) & 5450 (73.5\%) & 2265 (77.9\%) & $<0.001$ & $6090(74.3 \%)$ & $615(76.2 \%)$ & 1010 (76.3\%) & 0.118 \\
\hline & $\mathrm{G} 3 / 4$ & 2610 (25.3\%) & 1969 (26.5\%) & $641(22.1 \%)$ & & 2105 (25.7\%) & $192(23.8 \%)$ & $313(23.7 \%)$ & \\
\hline Chemo- and/or & No & $2842(27.5 \%)$ & 2294 (30.9\%) & $548(18.9 \%)$ & $<0.001$ & $2216(27.0 \%)$ & 235 (29.1\%) & $391(29.6 \%)$ & 0.437 \\
\hline Radiotherapy & Yes & 7483 (72.5\%) & 5125 (69.1\%) & 2358 (81.1\%) & & 5979 (73.0\%) & 572 (70.9\%) & 932 (70.4\%) & \\
\hline \multirow[t]{2}{*}{ Year of diagnosis } & 2010-2012 & 5304 (51.4\%) & 3915 (52.8\%) & 1389 (47.8\%) & $<0.001$ & 4279 (52.2\%) & $365(45.2 \%)$ & $660(49.9 \%)$ & 0.005 \\
\hline & 2013-2015 & $5021(48.6 \%)$ & 3504 (47.2\%) & 1517 (52.2\%) & & 3916 (47.8\%) & $442(54.8 \%)$ & $663(50.1 \%)$ & \\
\hline \multirow[t]{4}{*}{ Age (years) } & $<50$ & 1799 (17.4\%) & 1130 (15.2\%) & 669 (23.0\%) & $<0.001$ & 1474 (18.0\%) & $120(14.9 \%)$ & $205(15.5 \%)$ & 0.005 \\
\hline & $50-64$ & 4065 (39.4\%) & 2843 (38.3\%) & 1222 (42.1\%) & & 3241 (39.5\%) & 285 (35.3\%) & $539(40.7 \%)$ & \\
\hline & $65-79$ & 3247 (31.4\%) & 2448 (33.0\%) & 799 (27.5\%) & & 2525 (30.8\%) & 285 (35.3\%) & 437 (33.0\%) & \\
\hline & $80+$ & $1214(11.8 \%)$ & $998(13.5 \%)$ & $216(7.4 \%)$ & & 955 (11.7\%) & $117(14.5 \%)$ & $142(10.7 \%)$ & \\
\hline \multirow[t]{2}{*}{ Gender } & Male & 5693 (55.1\%) & 4159 (56.1\%) & 1534 (52.8\%) & 0.003 & 4585 (55.9\%) & 387 (48.0\%) & $721(54.5 \%)$ & 0.003 \\
\hline & Female & 4632 (44.9\%) & 3260 (43.9\%) & 1372 (47.2\%) & & 3610 (44.1\%) & $420(52.0 \%)$ & $602(45.5 \%)$ & \\
\hline \multirow[t]{3}{*}{ Ethnicity } & Caucasian & 7787 (75.4\%) & 5576 (75.2\%) & $2211(76.1 \%)$ & 0.336 & $6237(76.1 \%)$ & $591(73.2 \%)$ & $959(72.5 \%)$ & 0.117 \\
\hline & African-American & 1555 (15.1\%) & 1117 (15.1\%) & $438(15.1 \%)$ & & 1219 (14.9\%) & $115(14.3 \%)$ & $221(16.7 \%)$ & \\
\hline & Other/Unknown & $983(9.5 \%)$ & 726 (9.8\%) & $257(8.8 \%)$ & & $739(9.0 \%)$ & $101(12.5 \%)$ & $143(10.8 \%)$ & \\
\hline \multirow[t]{3}{*}{ Marital status } & Married & $5583(54.1 \%)$ & 3925 (52.9\%) & $1658(57.1 \%)$ & $<0.001$ & 4501 (54.9\%) & $399(49.4 \%)$ & $683(51.6 \%)$ & 0.061 \\
\hline & Single/Widowed & 3057 (29.6\%) & 2256 (30.4\%) & $801(27.6 \%)$ & & $2398(29.3 \%)$ & $259(32.1 \%)$ & $400(30.2 \%)$ & \\
\hline & Other/Unknown & 1685 (16.3\%) & 1238 (16.7\%) & $447(15.4 \%)$ & & 1296 (15.8\%) & $149(18.5 \%)$ & $240(18.1 \%)$ & \\
\hline
\end{tabular}

$\mathrm{n}$ (percent)

${ }^{\mathrm{a} C h i}$-squared test

logistic regression confirmed the significant imbalances in the baseline characteristics between patients with and without metastasectomy for the entire risk set except for the site of the primary, T-stage and ethnicity.

Impact of metastasectomy on survival for all metastatic sites The median CSS in patients with and without metastasectomy was 2.8 and 1.8 years and the 3-year survival rates were $46.3 \%$ (95\% confidence interval (CI): $44.1-$ $48.7 \%$ ) and $29.4 \%$ (95\% CI: $28.1-30.7 \%, \mathrm{HR}=0.62,95 \%$
CI: $0.58-0.66, P<0.001)$, respectively. The median OS in patients with and without metastasectomy was 2.6 and 1.7 years and the 3-year survival rates were $44.5 \%$ (95\% CI: $42.3-46.8 \%$ ) and $27.5 \%$ (95\% CI: $26.2-28.7 \%, \mathrm{HR}=$ $0.62,95 \%$ CI: $0.58-0.65, P<0.001)$, respectively. In multivariable analysis, metastasectomy was associated with improved CSS (HR $=0.75,95 \%$ CI: $0.70-0.80, P<$ $0.001)$ and OS (HR $=0.75,95 \%$ CI: $0.70-0.80, P<0.001$ ). Survival was better when the metastatic site was in the lung only and worse when occurring on both liver and 
lung data (Table 2). Survival was better in left sided colonic and rectal cancer, in nodal negative patients with lower $\mathrm{T}$-stage and lower graded tumors and in younger, married, Caucasian patients who underwent chemotherapy (Table 2).

\section{Impact of metastasectomy on survival stratified for the metastatic sites}

Metastasectomy was performed in 2500 of 8195 (30.5\%) patients with exclusively liver metastases, in 144 of 807
(17.8\%) patients with exclusively lung metastases and in 262 of $1323(19.8 \%)$ patients with metastases on both sites. Figure 3 summarizes the impact of metastasectomy for OS and CSS in stratified analyses performed separately for the three metastatic site groups.

\section{Resection of liver metastases only}

The stratified analysis demonstrated liver being the only metastatic site, for which metastasectomy was uniformly

Table 2 Prognostic factors for overall and cancer-specific survival

\begin{tabular}{|c|c|c|c|c|c|c|c|c|c|}
\hline & & \multicolumn{4}{|c|}{ overall survival using Cox regression } & \multicolumn{4}{|c|}{ cancer-specific survival using Cox regression } \\
\hline & & \multicolumn{2}{|l|}{ unadjusted $^{\mathrm{a}}$} & \multicolumn{2}{|l|}{ full model ${ }^{b}$} & \multicolumn{2}{|l|}{ unadjusted $^{a}$} & \multicolumn{2}{|l|}{ full model ${ }^{b}$} \\
\hline & & $\mathrm{HR}(95 \% \mathrm{Cl})$ & $p^{c}$ & $\mathrm{HR}(95 \% \mathrm{Cl})$ & $p^{c}$ & $\mathrm{HR}(95 \% \mathrm{Cl})$ & $p^{c}$ & $\mathrm{HR}(95 \% \mathrm{Cl})$ & $p^{c}$ \\
\hline \multirow[t]{2}{*}{ Resection } & No & Reference & $<0.001$ & Reference & $<0.001$ & Reference & $<0.001$ & Reference & $<0.001$ \\
\hline & Yes & $0.62(0.58-0.65)$ & & $0.75(0.70-0.80)$ & & $0.62(0.58-0.66)$ & & $0.75(0.70-0.80)$ & \\
\hline \multirow[t]{3}{*}{ Metastasis } & Liver only & Reference & $<0.001$ & Reference & $<0.001$ & Reference & $<0.001$ & Reference & $<0.001$ \\
\hline & Lung only & $0.78(0.70-0.86)$ & & $0.69(0.62-0.76)$ & & $0.75(0.68-0.84)$ & & $0.67(0.60-0.75)$ & \\
\hline & Liver and Lung & $1.62(1.51-1.74)$ & & $1.58(1.47-1.69)$ & & $1.64(1.52-1.76)$ & & $1.59(1.48-1.71)$ & \\
\hline \multirow[t]{5}{*}{ Tumor localization } & Right & Reference & $<0.001$ & Reference & $<0.001$ & Reference & $<0.001$ & Reference & $<0.001$ \\
\hline & Transverse & $0.91(0.84-0.98)$ & & 1.01 (0.93-1.09) & & $0.91(0.84-0.98)$ & & $1.01(0.93-1.10)$ & \\
\hline & Left & $0.61(0.57-0.65)$ & & $0.77(0.72-0.82)$ & & $0.61(0.57-0.65)$ & & $0.77(0.72-0.82)$ & \\
\hline & Rectosigmoid & $0.58(0.53-0.64)$ & & $0.72(0.66-0.80)$ & & $0.58(0.53-0.64)$ & & $0.73(0.66-0.80)$ & \\
\hline & Rectum & $0.46(0.42-0.50)$ & & $0.75(0.68-0.83)$ & & $0.46(0.41-0.50)$ & & $0.75(0.68-0.83)$ & \\
\hline \multirow[t]{2}{*}{ T-Stage } & T1 to T3 & Reference & $<0.001$ & Reference & $<0.001$ & Reference & $<0.001$ & Reference & $<0.001$ \\
\hline & $\mathrm{T} 4$ & $1.60(1.52-1.69)$ & & $1.44(1.37-1.52)$ & & $1.74(1.62-1.87)$ & & $1.47(1.40-1.56)$ & \\
\hline \multirow[t]{2}{*}{ N-Stage } & NO & Reference & $<0.001$ & Reference & $<0.001$ & Reference & $<0.001$ & Reference & $<0.001$ \\
\hline & N1 & $1.64(1.54-1.76)$ & & 1.57 (1.47-1.69) & & $1.74(1.62-1.87)$ & & $1.65(1.53-1.78)$ & \\
\hline \multirow[t]{2}{*}{ Grade } & $\mathrm{G} 1 / 2$ & Reference & $<0.001$ & Reference & $<0.001$ & Reference & $<0.001$ & Reference & $<0.001$ \\
\hline & $\mathrm{G} 3 / 4$ & $1.73(1.63-1.82)$ & & $1.50(1.42-1.59)$ & & 1.77 (1.67-1.87) & & $1.53(1.44-1.62)$ & \\
\hline Chemo- and/or & No & Reference & $<0.001$ & Reference & $<0.001$ & Reference & $<0.001$ & Reference & $<0.001$ \\
\hline Radiotherapy & Yes & $0.29(0.27-0.30)$ & & $0.34(0.32-0.36)$ & & $0.30(0.28-0.31)$ & & $0.35(0.33-0.37)$ & \\
\hline \multirow[t]{2}{*}{ Year of diagnosis } & 2010-2012 & Reference & $<0.001$ & Reference & 0.044 & Reference & $<0.001$ & Reference & 0.052 \\
\hline & 2013-2015 & $0.89(0.84-0.94)$ & & $0.94(0.89-1.00)$ & & 0.89 (0.84-0.94) & & $0.94(0.89-1.00)$ & \\
\hline \multirow[t]{4}{*}{ Age (years) } & $<50$ & Reference & $<0.001$ & Reference & $<0.001$ & Reference & $<0.001$ & Reference & $<0.001$ \\
\hline & $50-64$ & $1.24(1.14-1.34)$ & & $1.12(1.03-1.21)$ & & $1.21(1.12-1.31)$ & & $1.10(1.01-1.19)$ & \\
\hline & $65-79$ & $1.71(1.58-1.85)$ & & $1.35(1.25-1.47)$ & & $1.62(1.50-1.76)$ & & $1.29(1.19-1.41)$ & \\
\hline & $80+$ & $3.59(3.28-3.94)$ & & $1.96(1.77-2.17)$ & & $3.35(3.05-3.69)$ & & $1.85(1.67-2.05)$ & \\
\hline \multirow[t]{2}{*}{ Gender } & male & Reference & 0.106 & Reference & 0.048 & Reference & 0.034 & Reference & 0.2227 \\
\hline & female & $1.04(0.99-1.10)$ & & $0.95(0.90-1.00)$ & & $1.06(1.00-1.12)$ & & $0.97(0.92-1.02)$ & \\
\hline \multirow[t]{3}{*}{ Ethnicity } & Caucasian & Reference & $<0.001$ & Reference & $<0.001$ & Reference & $<0.001$ & Reference & 0.001 \\
\hline & African-American & $1.15(1.08-1.24)$ & & $1.11(1.04-1.20)$ & & $1.15(1.08-1.24)$ & & $1.11(1.03-1.20)$ & \\
\hline & other/unknown & $0.91(0.83-1.00)$ & & $0.91(0.83-0.99)$ & & $0.90(0.82-0.99)$ & & $0.89(0.81-0.98)$ & \\
\hline \multirow[t]{3}{*}{ Marital status } & married & Reference & $<0.001$ & Reference & $<0.001$ & Reference & $<0.001$ & Reference & $<0.001$ \\
\hline & single & $1.46(1.38-1.55)$ & & $1.21(1.14-1.28)$ & & $1.45(1.36-1.53)$ & & $1.20(1.13-1.28)$ & \\
\hline & other/unknown & $1.12(1.05-1.21)$ & & $1.05(0.98-1.13)$ & & 1.09 (1.01-1.17) & & $1.02(0.94-1.10)$ & \\
\hline
\end{tabular}


beneficial regarding OS and CSS in unadjusted and all adjusted analyses (Figs. 2 and 3).

\section{Resection of lung metastases only}

For lung as the only metastatic site, metastasectomy was beneficial only in unadjusted analysis. The median OS in patients with and without lung metastasectomy was 3.3 and 2.5 years and the 3-year survival rates were $58.0 \%$ (95\% CI: 49.1-68.4\%) and 40.9\% (95\% CI: 36.3-46.1\%) (HR $=0.73,95 \%$ CI: 0.56-0.95, $P=0.016)$. However, after multivariable adjustment, lung metastasectomy did not have a significant impact on OS $(\mathrm{HR}=0.86,95 \% \mathrm{CI}$ : $0.65-1.14, P=0.280)$ and CSS $(\mathrm{HR}=0.84,95 \% \mathrm{CI}: 0.62-$ $1.12, P=0.232$ ). Furthermore, no benefit on CSS and OS was found in patients undergoing resection of lung metastases after propensity score- (PS) and inverse probability weighted- (IPW) adjustment (Figs. 2 and 3).

\section{Resection of combined lung and liver metastases}

In patients with both lung and liver metastases, metastasectomy was beneficial only in unadjusted analysis. The median overall survivals in patients with and without metastasectomy were 1.5 and 1.2 years. However, none of the adjusted analyses yielded a survival benefit for patients undergoing metastasectomy (Figs. 2 and 3).

\section{Discussion}

This is the first SEER analysis using IPW and PS to assess the impact of metastasectomy on survival in colorectal cancer patients with special focus on liver and/or lung metastases and with removed primary tumor. The present analysis provides compelling evidence of a statistically significant and clinically relevant increase in OS and CSS for liver resection but not for metastasectomy of lung or both sites.

Outcomes of patients with metastatic colorectal cancer ( $\mathrm{mCRC}$ ) have improved enormously over the last decade. Indeed, depending on the extent of metastases and the biology, the median OS of metastatic colorectal cancer patients can exceed 3 years [27]. There are different factors, which led to improved outcomes in patients with metastatic colorectal cancer: first, our knowledge of the tumor heterogeneity based on molecular profiling has changed the therapeutic management of these tumors. Thus, the more individual systemic treatment results in higher response rates and consecutively higher rates of surgical metastasectomy. These therapeutic concepts are well approved in large randomized trials in the first line as well second line settings [11, 28]. As refractory patients will be seen more frequently in sequential treatment of mCRC re-challenge concepts have been investigated with promising results $[29,30]$.

However, the therapeutic concepts of mCRC do not only contain chemotherapy or antibodies. Resections of oligometastatic liver and or lung metastasis are commonly discussed during multidisciplinary tumor boards and up to $15 \%$ of mCRC patients are evaluated for resection. The surgical management along the current guidelines of

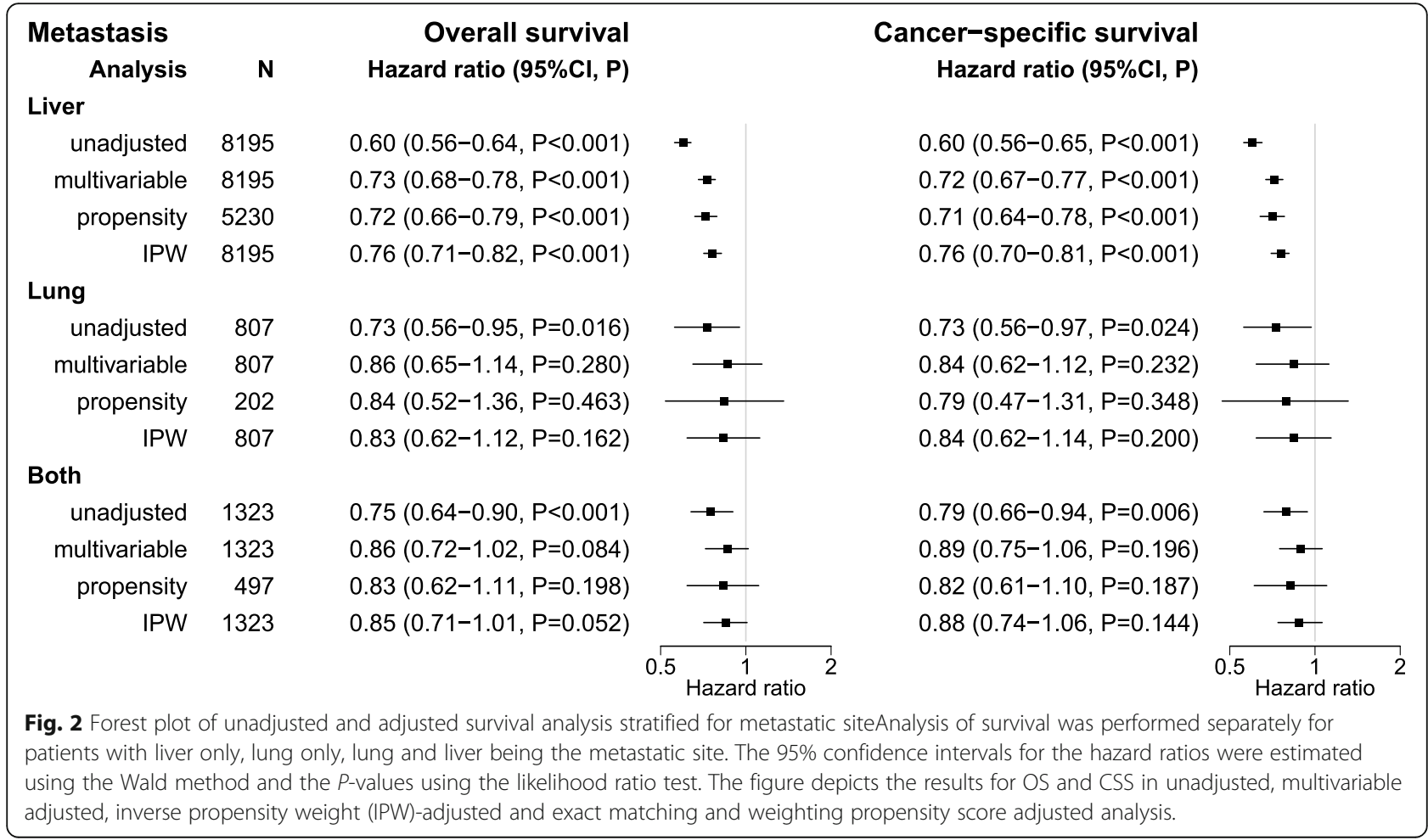




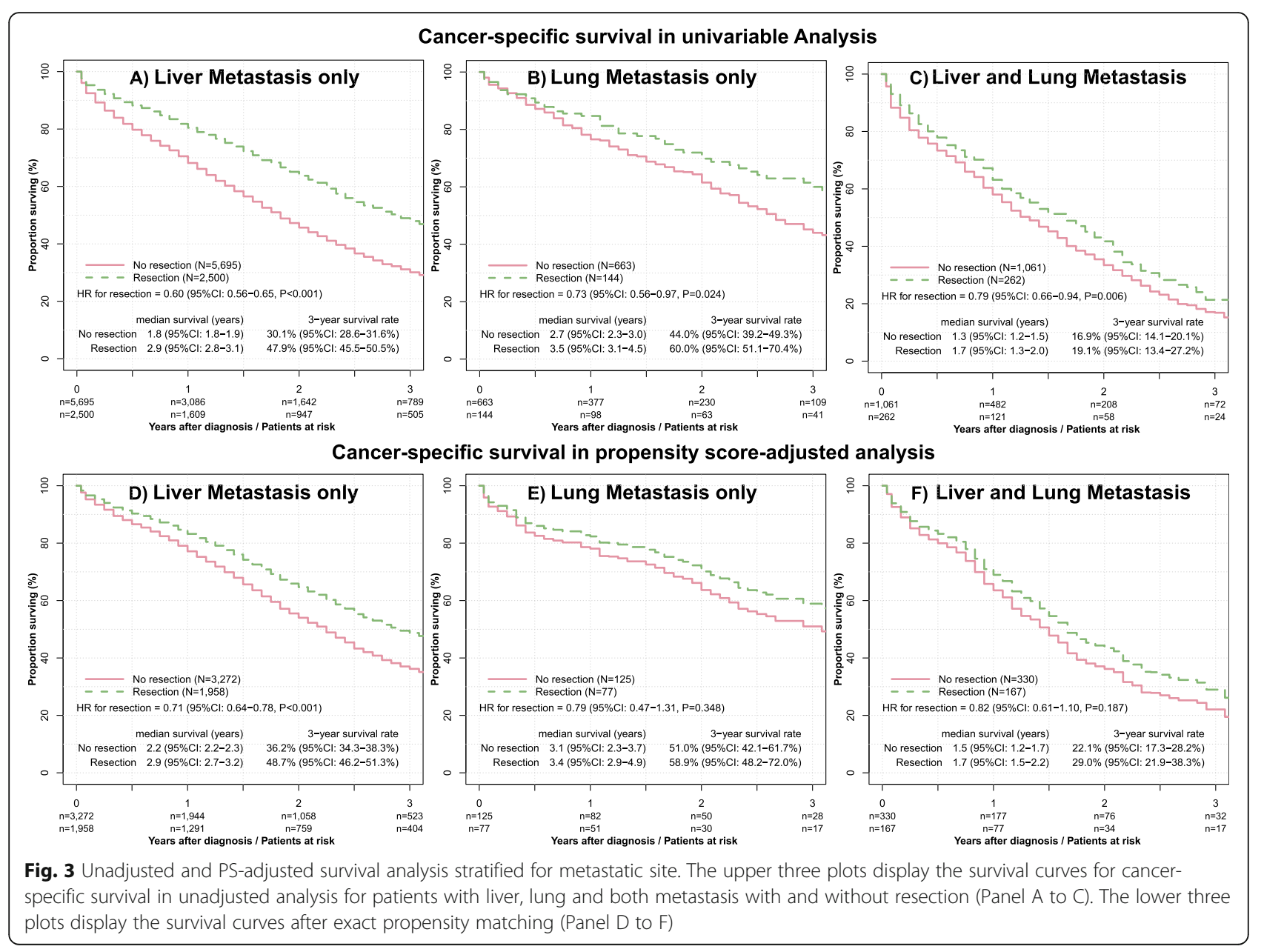

National Comprehensive Cancer Network (NCCN) and European Society for Medical Oncology (ESMO) is not restricted to one single organ as well no clear definition on the number of metastasis at liver or lung will restrict such procedures within a multidisciplinary approach to mCRC patients $[17,18]$. However, good prognostic and predictive makers to guide this decision are still missing.

The benefit of metastatic resection for these selected patients led to an improvement of 5-years survival of 20-38\% [31-33]. Most available data supporting resection of liver and or lung metastases are based on small case series as well retrospective data and reviews [34, 35]. Large cohort analyses to address the impact of metastasectomy in relation to OS or CSS are scarse.

To our knowledge this is the first population-based, propensity score adjusted analysis investigating the prognostic impact of resection of liver and or lung metastases in mCRC patients. Being aware of the conflicting data and the challenge to handle relevant bias due to substantial imbalances between resected versus not resected mCRC patients, we have intentionally selected the propensity score matching as a superior and refined statistical method in addition to commonly used multivariate analysis.

Based on a large collective from the SEER database of patients with metastatic colorectal cancer between 2010 and 2015 with resected primary tumor, the present study provides compelling evidence that the prognosis of patients with resection of liver metastasis in the overall population is better after adjusting for a strong bias regarding various patient and tumor characteristics by the use of the propensity score matching. Conversely, no differences in OS and CSS appeared in the propensity score adjusted population for the patients undergoing resection of lung metastases or both lung and liver metastases. Thus, we conclude that the overall survival improvement in $\mathrm{mCRC}$ patients after resection of lung and both lung and liver metastases described in the scientific literature are not real on a population-based level but caused by differences regarding confounders that could not be adjusted for in multivariate regression analysis.

Our results differ from the finding from Boysen et al. [36] demonstrating a survival benefit of lung metastasectomy 
compared to the group of no resection. However, in this Danish cohort study the benefit for lung metastasectomy was only found in univariate analyses and non-significant benefit was seen at their multivariate analysis for lung metastasectomy, hence selection bias (healthier patients with less metastatic disease, and better biology get operated) is inherent. Luo et colleagues [37] demonstrated in their SEER database analysis that the metastatic site of patients with $\mathrm{mCRC}$ has prognostic impact. Indeed, isolated liver metastases have a better prognosis compared to metastases at multiple sites. However, the authors did not analyze the impact on outcomes of resection and survival of mCRC patients.

In highly selected patients there might be a benefit for a sequential metastasectomy of liver followed by systemic treatment followed by metastasectomy of the lung. However, the patient numbers in published analyses are low and without control group [38-42]. The same trend of survival benefit was reported for patients with mCRC after lung resection [43-45]. The limitations of these studies are the retrospective nature, inclusion of a highly selective patient cohort and most of them were done at a highly specialized cancer center and hence lack the generalizability to other hospitals. Most importantly, no thorough risk-adjustment was performed with propensityscore analyses and hence relevant selection bias is an inherent shortcoming. This explains the different results compared to our study, in which we aimed to properly address and limit selection bias.

Our study has some limitations, most of them by the lack of information which were not available from the SEER database. In fact, the SEER database does not provide any information about the intention or the extend of metastasectomy. Hence, we can not ascertain if all the three groups underwent metastasectomy with curative intention. However, there is some evidence that resection of lung metastasis in the setting of liver and lung metastasis in mCRC does not improve the survival [46], which is in accordance to our findings.

One limitation is the lack of biomarker information regarding RAS and rapidly accelerated fibrosarcoma isoform B (BRAF) mutations as well as microsatellite instability. Second, no information concerning the choice of systemic therapy as well the status of conversion rate for resection of initially unresectable metastasis are available. Third, information regarding the time-point of resection, type of surgery as well the resection margin ( $R 0$ or R1) was lacking. Fourth, the SEER database does not provide any information regarding the diagnosis of pulmonary metastasis. Hence it is possible, that small pulmonary nodules in computed tomography (CT) scans were benign. However, due to the population-based nature of this analysis that reflects the real United States population with metastatic colon cancer, the lack of this information does not impact our results, albeit limits the extent of interpretation of our data.

\section{Conclusion}

This population-based propensity score adjusted analysis of mCRC patients with liver and or lung metastases provides compelling evidence that the resection of liver metastases improves OS and CSS. In contrary, the resection lung metastases as well as both lung and liver metastases did not result in increased survival.

\begin{abstract}
Abbreviations
BRAF: Rapidly accelerated fibrosarcoma isoform B; Cl: Confidence interval; CRC: Colorectal cancer; CRLM: Colorectal liver metastases; CSS: Cancer specific survival; CT: Computed tomography; EGFR: Epidermal growth factor; ESMO: European Society for Medical Network; HR: Hazard ratio; IPW: Inverse probability weighted; mCRC: metastatic colorectal cancer; NCCN: National Comprehensive Cancer Network; OS: Overall survival; PS: Propensity score; RAF: Rapidly accelerated fibrosarcoma; RAS: Rat sarcoma; SEER: Surveillance epidemiology and end results database
\end{abstract}

\section{Acknowledgements}

Not applicable

\section{Authors' contributions}

ARS participated in interpretation of the data analysis, conducting literature search, figures, and drafting, writing, and critical reviewing of the manuscript. UG participated in study design, interpretation of the data analysis, literature search, figures, and writing and critical reviewing of manuscript. RW participated in study design, performing data analysis, interpretation of the data analysis, literature search, and manuscript writing and critical reviewing. All authors read and approved the final manuscript.

\section{Funding}

This study was not funded by any source.

\section{Availability of data and materials}

The data used in this study are available free of charge online at www.seer. cancer.gov on request.

All data generated or analyzed during this study are included in this published article.

\section{Ethics approval and consent to participate}

This study was based on public use de-identified data from the SEER database and did not involve interaction with human subjects or use personal identifying information. The study did not require informed consent from the SEER registered cases and the authors obtained Limited-Use Data Agreements from SEER. No trial registration was necessary.

\section{Consent for publication}

Not applicable

\section{Competing interests}

The authors declared that they have no competing interest. No third-party financial funds or materials were accepted or necessary for execution of this research project.

\section{Author details}

${ }^{1}$ Clinic for Medical Oncology and Hematology, University Hospital Zurich and University of Zurich, CH-8091 Zürich, Switzerland. ${ }^{2}$ University Clinic for Visceral Surgery and Medicine, University Hospital Berne, CH-3010 Berne, Switzerland. ${ }^{3}$ Onkologie und Hämatologiezentrum Stial STS AG, CH-3600 Thun, Switzerland. ${ }^{4}$ Division of Medical Oncology and Hematology, Kantonsspital St. Gallen, CH-9007 St. Gallen, Switzerland. ${ }^{5}$ Department of Surgery, Kantonsspital St. Gallen, CH-9007 St. Gallen, Switzerland. Institute of Medical Biometry and Informatics, University Heidelberg, 69120 Heidelberg, Germany. 
Received: 30 December 2019 Accepted: 3 March 2020

Published online: 23 March 2020

\section{References}

1. Cronin KA, et al. Annual report to the nation on the status of cancer, part I: national cancer statistics. Cancer. 2018;124(13):2785-800.

2. Brenner H, Kloor M, Pox CP. Colorectal cancer. Lancet. 2014;383(9927): 1490-502.

3. Kune GA, et al. Survival in patients with large-bowel cancer. A populationbased investigation from the Melbourne colorectal cancer study. Dis Colon Rectum. 1990;33(11):938-46.

4. Manfredi S, et al. Epidemiology and management of liver metastases from colorectal cancer. Ann Surg. 2006;244(2):254-9.

5. van der Geest LG, et al. Nationwide trends in incidence, treatment and survival of colorectal cancer patients with synchronous metastases. Clin Exp Metastasis. 2015;32(5):457-65.

6. Scheithauer W, et al. Randomised comparison of combination chemotherapy plus supportive care with supportive care alone in patients with metastatic colorectal cancer. BMJ. 1993;306(6880):752-5.

7. Geva R, et al. Retrospective analysis of efficacy and safety of third-line chemotherapy for metastatic colorectal cancer among elderly patients receiving targeted therapy in early lines. JCGG. 2015;6(3):95-99.

8. Stintzing $S$, et al. Impact of BRAF and RAS mutations on first-line efficacy of FOLFIRI plus cetuximab versus FOLFIRI plus bevacizumab: analysis of the FIRE-3 (AlO KRK-0306) study. Eur J Cancer. 2017;79:50-60.

9. Stintzing $\mathrm{S}$, et al. FOLFIRI plus cetuximab versus FOLFIRI plus bevacizumab for metastatic colorectal cancer (FIRE-3): a post-hoc analysis of tumour dynamics in the final RAS wild-type subgroup of this randomised openlabel phase 3 trial. Lancet Oncol. 2016;17(10):1426-34.

10. Elez E, Argiles G, Tabernero J. First-line treatment of metastatic colorectal cancer: interpreting FIRE-3, PEAK, and CALGB/SWOG 80405. Curr Treat Options in Oncol. 2015;16(11):52.

11. Arnold D, et al. Prognostic and predictive value of primary tumour side in patients with RAS wild-type metastatic colorectal cancer treated with chemotherapy and EGFR directed antibodies in six randomized trials. Ann Oncol. 2017;28(8):1713-29.

12. Kroemer $\mathrm{G}$, et al. Colorectal cancer: the first neoplasia found to be under immunosurveillance and the last one to respond to immunotherapy? Oncoimmunology. 2015;4(7):e1058597.

13. Saltzstein SL, Behling CA. Age and time as factors in the left-to-right shift of the subsite of colorectal adenocarcinoma: a study of 213,383 cases from the California cancer registry. J Clin Gastroenterol. 2007;41(2):173-7.

14. Elnatan J, Goh HS, Smith DR. C-KI-RAS activation and the biological behaviour of proximal and distal colonic adenocarcinomas. Eur J Cancer. 1996;32A(3):491-7.

15. Benedix F, et al. Comparison of 17,641 patients with right- and left-sided colon cancer: differences in epidemiology, perioperative course, histology, and survival. Dis Colon Rectum. 2010;53(1):57-64.

16. Clark S, Suttie SA, Shaikh I, Mullen R, et al. Outcome of right- and left-sided colonic and rectal cancer following surgical resection. Color Dis. 2011;13: 884-9 Colorectal Dis, 2012. 14(3): p. 386; author reply 386-7.

17. Yoshino T, et al. Pan-Asian adapted ESMO consensus guidelines for the management of patients with metastatic colorectal cancer: a JSMO-ESMO initiative endorsed by CSCO, KACO, MOS, SSO and TOS. Ann Oncol. 2018; 29(1):44-70

18. Van Cutsem $\mathrm{E}_{\text {, et }}$ al. ESMO consensus quidelines for the management of patients with metastatic colorectal cancer. Ann Oncol. 2016;27(8):1386-422.

19. Surveillance Epidemiology and End Results (SEER) Program, Research Data (National Cancer Institute, DCCPS Surveillance Research Program, Surveillance Systems Branch, released April 2015, based on the November 2014 submission. www.seer.cancer.gov, 1973-2012.

20. Grambsch P, Therneau T. Proportional hazards tests and diagnostics based on weighted residuals. Biometrika. 1994;81:515-26.

21. Noone AM, et al. Comparison of SEER treatment data with Medicare claims. Med Care. 2016;54(9):e55-64

22. van der Wal WM, Geskus RB. Ipw: an R package for inverse probability weighting. J Stat Softw. 2011:43:1.

23. Joffe M, Rosenbaum P. Invited commentary: propensity scores. Am J Epidemiol. 1999:150:327-33.

24. Rosenbaum P. Model-based direct adjustment. J Am Stat Assoc. 1987;82:387-94.
25. Rubin DB. Estimating causal effects from large data sets using propensity scores. Ann Intern Med. 1997;127(8 Pt 2):757-63.

26. Ho D, et al. Matching as nonparametric preprocessing for reducing model dependence in parametric causal inference. Polit Anal. 2007:15:199-236.

27. Prasanna T, et al. The survival outcome of patients with metastatic colorectal cancer based on the site of metastases and the impact of molecular markers and site of primary cancer on metastatic pattern. Acta Oncol. 2018;57:1-7.

28. Siravegna $\mathrm{G}$, et al. Clonal evolution and resistance to EGFR blockade in the blood of colorectal cancer patients. Nat Med. 2015;21(7):795-801.

29. Vogel A, et al. Treatment decisions in metastatic colorectal cancer - beyond first and second line combination therapies. Cancer Treat Rev. 2017:59:54-60

30. Goldberg RM, et al. Optimising the use of cetuximab in the continuum of care for patients with metastatic colorectal cancer. ESMO Open. 2018;3(4): e000353.

31. Choti MA, et al. Trends in long-term survival following liver resection for hepatic colorectal metastases. Ann Surg. 2002;235(6):759-66.

32. Pawlik TM, et al. Effect of surgical margin status on survival and site of recurrence after hepatic resection for colorectal metastases. Ann Surg. 2005: 241(5):715-22 discussion 722-4.

33. Kanas GP, et al. Survival after liver resection in metastatic colorectal cancer: review and meta-analysis of prognostic factors. Clin Epidemiol. 2012:4:283-301.

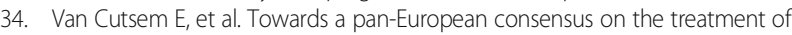
patients with colorectal liver metastases. Eur J Cancer. 2006:42(14):2212-21.

35. Kemeny N. Management of liver metastases from colorectal cancer. Oncology (Williston Park). 2006;20(10):1161-76 1179; discussion 1179-80, 1185-6.

36. Boysen AK, et al. Metastasis directed therapy for liver and lung metastases from colorectal cancer - a population based study. Int J Cancer. 2018; 143(12):3218.

37. Luo D, et al. Prognostic value of distant metastasis sites and surgery in stage IV colorectal cancer: a population-based study. Int J Color Dis. 2018; 33(9):1241.

38. Brouquet A, et al. Improved survival after resection of liver and lung colorectal metastases compared with liver-only metastases: a study of 112 patients with limited lung metastatic disease. J Am Coll Surg. 2011;213(1): 62-9 discussion 69-71.

39. Hadden WJ, et al. Resection of colorectal liver metastases and extra-hepatic disease: a systematic review and proportional meta-analysis of survival outcomes. HPB (Oxford). 2016;18(3):209-20.

40. Headrick JR, et al. Surgical treatment of hepatic and pulmonary metastases from colon cancer. Ann Thorac Surg. 2001;71(3):975-9 discussion 979-80.

41. Marin C, et al. Outcome of strict patient selection for surgical treatment of hepatic and pulmonary metastases from colorectal cancer. Dis Colon Rectum. 2013;56(1):43-50.

42. Pulitano $\mathrm{C}$, et al. Liver resection for colorectal metastases in presence of extrahepatic disease: results from an international multi-institutional analysis. Ann Surg Oncol. 2011:18(5):1380-8.

43. Gonzalez M, et al. Risk factors for survival after lung metastasectomy in colorectal cancer patients: a systematic review and meta-analysis. Ann Surg Oncol. 2013;20(2):572-9.

44. Gonzalez M, Gervaz P. Risk factors for survival after lung metastasectomy in colorectal cancer patients: systematic review and meta-analysis. Future Oncol. 2015;11(2 Suppl):31-3.

45. $\mathrm{Xu} \mathrm{L}$, et al. Parallel calibration revisited: the second direction for shrinkage estimation of regression coefficients can be as natural and necessary as the traditional one. Anal Chim Acta. 2009:644(1-2):25-9.

46. Patel $D$, et al. Is survival for patients with Resectable lung metastatic colorectal cancer comparable to those with Resectable liver disease? Results from the south Australian metastatic colorectal registry. Ann Surg Oncol. 2016:23(11):3616-22.

\section{Publisher's Note}

Springer Nature remains neutral with regard to jurisdictional claims in published maps and institutional affiliations. 